\title{
Spatial problem solving: The integration of independently learned cognitive maps
}

\author{
GERARD L. HANLEY and MARVIN LEVINE \\ State University of New York, Stony Brook, New York
}

\begin{abstract}
The process of mentally integrating separate regions of a layout into a unified representation is a critical component in generating inferred spatial information. Blindfolded subjects learned two different paths by tactual exploration. The subjects were then given information that permitted them to integrate the two cognitive maps of the paths into one map. In Experiment 1, the speed and accuracy of originally learned and inferred movements within a path were equivalent, whereas inferred movements between the two paths were significantly slower and less accurate. In Experiment 2, the subjects could request additional training on the paths before they were given the integration information, and they also rated how well they knew the path on which they were tested. When the subjects judged that they remembered all or most of the path information, movements between two paths and within one path were performed with the same accuracy. The equivalent accuracy of all the movements suggests that cognitive integration can generate an accurate representation of an integrated layout when the path information is recalled.
\end{abstract}

Solving a spatial problem typically begins with learning a spatial layout of an environment and subsequently being tested on at least part of the environment. If the person exploring the space functioned as a cartographer, the terrain's spatial relationships would be translated into an external representation, that is, into a physical map. Downs (1981) discussed how the map and mapping concepts have been used as metaphors to model an individual translating an environment into an internal representation, that is, into a cognitive map. As did Siegel (1981), he cautioned against the reification of the cognitive map and mapping concepts. We agree with this position and will use these terms as guiding metaphors.

Two types of cognitive maps have been distinguished in the literature: route maps and survey, or configural, maps (Chase \& Chi, 1980; Downs, 1981; Levine, Jankovic, \& Palij, 1982; Siegel, 1981; Siegel \& White, 1975). Route maps preserve both the ordinal position in which the landmarks are perceived while a subject is traveling along a path and the distance between successive landmarks (Siegel, Kirasic, \& Kail, 1978). For example, the spatial layouts shown in Figure 1 represent two different routes through the same environment. Points $\mathrm{A}$ and $\mathrm{D}$ would be the most distant points

Support for the research described here was provided by National Science Foundation Grant BNS78-24716, awarded to the second author, and by research grants-in-aid from Sigma Xi, the Scientific Research Society, awarded to the first author. Portions of this article were presented at the annual meetings of the Eastern Psychological Association, April 1980. We would like to thank Marcia K. Johnson, Charles Clifton, and the anonymous reviewers for their valuable comments on earlier drafts. Our mailing address is: Department of Psychology, State University of New York, Stony Brook, New York 11794. in the route map of the path shown in Figure 1a. Although the four points are in the same relative locations in Figure $1 b$, the route map of the A-D-B-C path would represent Points $A$ and $D$ as the closest points. Thus, route knowledge is limited to the sequence of spatial relationships learned.

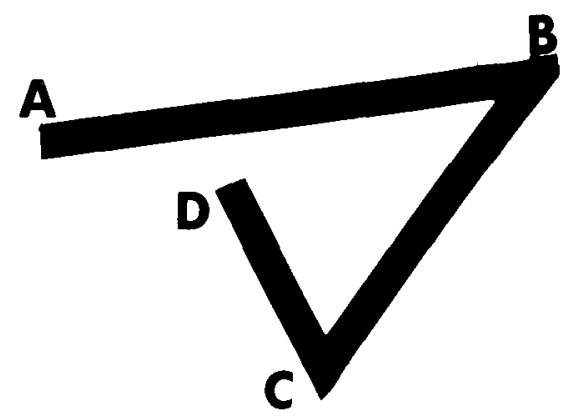

(1a) Route $A-B-C-D$

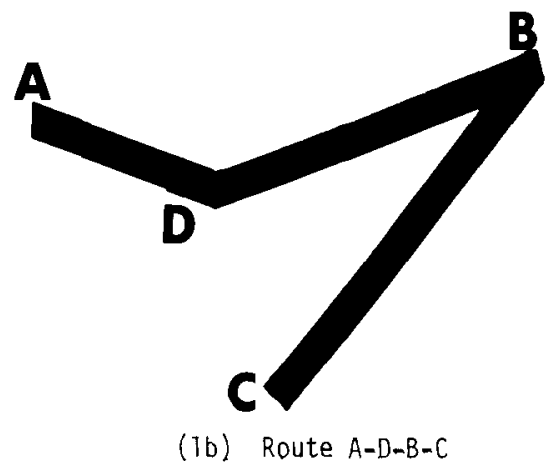

Figure 1. An example of two different routes through the same environment. 
The second class of cognitive mapping processes corresponds to the cartographic procedure of survey mapping. All spatial relationships between all locations are represented within a person's survey knowledge of an environment, and these relationships are represented simultaneously (Appleyard, 1970; Downs, 1981; Downs \& Stea, 1973; Levine, Jankovic, \& Palij, 1982; Lynch, 1960; Siegel \& White, 1975; Tolman, 1948; Shemyakin, Note 1). Levine et al. proposed that when the path to be learned is simple and is well learned, the cognitive map will be of the survey type. All the points will be represented in their mutual relationships. In other words, the cognitive map will be picturelike.

To realize the implications of this, consider the following concrete case. Suppose a blindfolded person has his or her finger traced through a four-point path, like the one in Figure 1a. The person will learn not only to move from $\mathrm{A}$ to $\mathrm{B}, \mathrm{B}$ to $\mathrm{C}$, and $\mathrm{C}$ to $\mathrm{D}$ (this would be route learning). Because all the points are simultaneously available in a picturelike representation, the person could now take shortcuts, that is, could move from any point to any other point. Furthermore, according to Levine et al., people will perform equally well whether the test consists of a learned movement (e.g., going from Point $C$ to Point D) or an inferred, shortcut movement (e.g., going from Point $\mathrm{C}$ to Point A). They demonstrated that this was the case for simple four- and fivepoint paths and called this the principle of equiavailability.

A consequence of the equiavailability principle is that the survey representation of a layout should not be affected by the different routes a person may have taken to explore the terrain, because all the relationships between locations, whether inferred or learned, would be represented equally well. For example, in Figures $1 \mathrm{a}$ and $1 \mathrm{~b}$, the distance and direction between Points A and B would be the same and would be represented with equal accuracy, because the different paths through the space would produce the same survey knowledge.

In the Levine et al. (1982) experiments, the subjects traversed the layouts of large-scale environments (environments in which all locations cannot be perceived from a single vantage point) in a single sequence. Typically, large-scale environments are large in size and people often do not have the opportunity to travel to all locations during a single trip. People may learn the spatial layout of a large-scale environment by selectively exploring separate regions of the space at different points in time. The present experiments examined the equiavailability principle under conditons in which people learned two regions of the environment separately and were required to cognitively integrate the cognitive maps of these two regions when suitable information was presented. The methodology was an extension of the finger-tracing procedure used by Levine et al., which modeled the learning of a large-scale environment in a small-sized space. In brief, subjects had their fingers traced over two spatially unrelated paths. That is, the paths the subjects learned were components of a larger layout, but there was no information during the initial training to indicate the spatial relationship between the component paths. Subjects were then given the information that spatially related the paths, thus creating an opportunity to integrate the two cognitive maps into one map.

If survey knowledge is not affected by the independent learning of the paths, and if subjects are able to integrate the paths into a single simultaneous representation, then subjects should be able to take shortcuts between the paths. They should be able to go from one point on one path to another point on the other path along an inferred route. The inferred route between paths should be as accurate as movements along an inferred or originally learned route within one path. The alternative hypothesis is that survey knowledge of the integrated path is not independent of the way the layout was explored. The component paths could be simultaneously but separately represented along with the additional information that spatially related the paths. Relationships between two points in separate paths would not be as available as routes within one path. Consequently, between-path movements would not be as accurate as movements (learned or inferred) within one path.

\section{EXPERIMENT 1}

\section{Method}

Subjects. Twelve right-handed undergraduates from the State University of New York at Stony Brook fulfilled a course requirement by participating in the experiment.

Stimuli. Each problem required the construction of a threepart display (see Figure 2): the integrated (or test) path in the center and its two components (the training paths) on either side. Each of the two training components contained three points, labeled 1-2-3 and A-B-C, respectively. The component paths were separated by approximately $600 \mathrm{~mm}$. The test path was constructed from the two training paths by defining an integration point, that is, by placing one point from each component onto the identical location. In Figure 2, for example, the test path is in the center, with Point $A$ in the identical place as Point $2(\mathrm{~A}=2)$. The average length of a single training path was $91 \mathrm{~mm}$, with a range of 75 to $125 \mathrm{~mm}$. The angle formed by the three points varied from 20 to $150 \mathrm{deg}$. Points 1 and $A$ were always to the right of Points 3 and $C$ on the numbered and lettered paths, respectively. The locations of Points $B$ and 2, however, were not restricted. Eighteen different sets of threepart displays were constructed. Test paths that looked like familiar forms, such as capital letters, were avoided.

Procedure. The subjects were run individually. The subjects sat at a table on which lay a picture of an integrated path and its two components (see Figure 2). This example was referred to during the instructions. The subjects were told that they would be blindfolded and would then have to learn two different paths; one path would be traced in a position to their left and the other to their right. After they had received a certain number of tracings over each path, a common point would be announced. The subjects were then to combine the two paths such that the two announced points were in the same location. They were instructed that the paths would not be rotated; the paths would have the same orientation as in the tracing pro- 


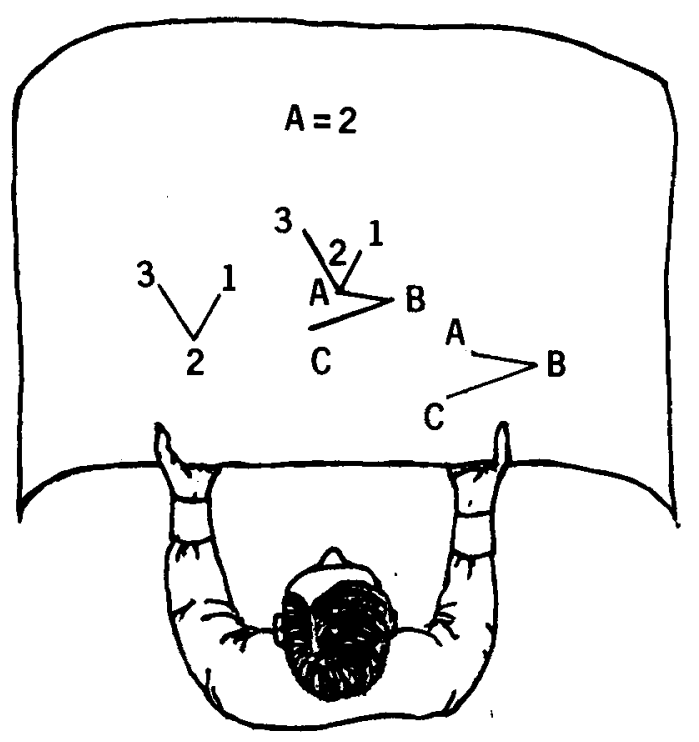

Figure 2. A three-part display depicting the tracing positions of the two component paths and (in the center) the testing position of the integrated path.

cedure. If they remembered the path configuration but forgot the labels, the subjects were instructed that they could identify the rightmost endpoints as being Points $A$ and 1 . Once they had combined the paths, they would have to draw a line directly from one point to another point. This sequence of path learning, path integration, and test constituted a single problem. The subjects received four practice problems and 54 experimental problems in the course of three sessions.

Training phase. The subject put on the blindfold and remained blindfolded throughout the entire session. The experimenter put a new display on the table, took the subject's right index finger, and traced it over each three-point path either two times (low training) or four times (high training). A tracing consisted of the following: The experimenter lifted the subject's finger from a starting position at the edge of the table, moved it to the first point on one of the paths, and announced that point (Point 1 or Point A). The experimenter then moved the finger along the path to the next point and announced that point and then moved it to the final point and announced that point. Each tracing over a path took about 3 sec. The subject's finger was returned to the starting position at the edge of the table for approximately $2 \mathrm{sec}$. The tracing procedure was then repeated on the same path. After the first path was traced twice, the second path was traced in the same manner, always with the subject's right index finger. The two tracings on each path constituted the low-training procedure. The high-training procedure consisted of repeating the low-training procedures. The first path and then the second path were again traced twice.

Once the training on both paths had been completed, the subject's finger was returned to the starting position. The integration point (e.g., $\mathrm{A}=2$ in Figure 2) was always immediately announced. If a subject requested it, the integration point was repeated. The subject was instructed to say "Ready" after cognitively integrating the paths. After giving this signal, the subject was tested.

Testing phase. The test took place on the integrated path directly in front of the subject. After learning the component paths, the subject (still blindfolded) was given a pencil, the tip of which was then placed on one point. That point was announced, and the subject was asked to draw a line directly, "as the crow flies," to another specified point. There were three types of tested relationships: a simple forward movement, a shortcut within one path, or a shortcut between two paths. A simple forward (SF) relationship was one segment of one of the originally learned path. It was tested by requiring the subject to move along the path, for example, from Point 1 to Point 2. A shortcut within one path (WSC) was an inferred spatial relationship between Points 1 and 3 or between Points A and C. SF and WSC were movements within one path. These are referred to here collectively as "within" movements. The third type of relationship was the shortcut between two paths (BSC). This "between" movement was an inferred spatial relationship, but it was between a point on one path and a point on the other path (excluding the integration point). In Figure 2, moving from Point B to Point 1 would be a test for this type of inferred relationship. If a subject wanted to repeat the tested movement, he or she could make one additional attempt to locate the target point. Only the subject's second movement was used in the data analysis. For all three types of tests, the distance between the tested points was always $50 \mathrm{~mm}$. The constant test distance eliminated possible confounds between the type of test and the speed and/or accuracy with which the movement was performed.

During the first session, the instructions and four practice problems were followed by 18 experimental problems. At the beginning of the second and third sessions, the experimenter briefly reviewed the procedure with the subject and then presented 18 experimental problems. Two to 3 days separated each 1 -h session. In each of the sessions, half of the problems entailed low training (two tracings on each component) and half entailed high training (four tracings). Three problems on which the subjects received no training on either path were randomly dispersed throughout the experiment. The subjects were only told the integration point and then were tested on a BSC relationship. The subjects never received any feedback, verbal or visual, about their performance throughout the entire experiment.

The subject's performance on each problem was assessed with three measures: two reaction times (RTs) and one responseaccuracy measure. The first was the thinking $\mathrm{RT}$. This was the clapsed time between the announcement of the integration points and the subject's ready signal. The drawing RT was the time between the announcement of the test movement ("You're at Point 1 , go to Point 3") and the completion of the movement. RTs were unobtrusively measured to the nearest second. Movement accuracy was assessed by the absolute angle deviation (angle error) of the drawn path from the correct test path.

Design. The three types of test movements occurred equally often with the two degrees of training within each session. Within every session, each of the nine intergration points $(A=1$, $A=2, \ldots, C=3$ ) occurred in the set of 9 high- and 9 lowtraining problems. The order in which the two component paths were traced (numbered path first vs. lettered path first) and the position on the tabletop in which the first path was traced (left position vs. right position) were counterbalanced. The subjects learned the same 18 integrated paths in each of the three sessions, but they were tested on a different type of relationship each time. The order and/or position of the tracing of the individual paths was different in each session. The order of problem presentation in each session was randomized.

\section{Results}

The data were analyzed using a repeated measures analysis of variance. The Newman-Keuls statistic was used to test the significance of all comparisons between conditions that were found to be significant by the overall analysis of variance. Two subjects each requested an additional attempt on one trial; one of the second attempts was more accurate and the other was less accurate than the first attempt.

Effects of type of movement. Table 1 shows the mean angle errors and drawing RTs for problems in which each type of movement was tested. There were significant differences in the angle errors and the draw. 
Table 1

The Means and Standard Deviations of Angle Errors (in Degrees) and Drawing Reaction Times (in Seconds) for the Three Test Movements

\begin{tabular}{cccccc}
\hline \multirow{2}{*}{$\begin{array}{c}\text { Test } \\
\text { Movement }\end{array}$} & \multicolumn{2}{c}{ Angle Error } & & \multicolumn{2}{c}{ Drawing RT } \\
\cline { 2 - 3 } \cline { 5 - 6 } & Mean & SD & & Mean & SD \\
\hline SF & 31.8 & 9.04 & 7.20 & 3.15 \\
WSC & 29.1 & 6.26 & 7.20 & 2.43 \\
BSC & 54.0 & 11.21 & & 12.90 & 6.48 \\
\hline
\end{tabular}

Note $-S F=$ simple forward; $W S C=$ shortcut within one path; $B S C=$ shortcut between two paths.

ing RTs among the movement types $[\mathrm{F}(2,22)=42.71$, $\mathrm{p}<.05$, and $\mathrm{F}(2,22)=17.55, \mathrm{p}<.05$, respectively] The amounts of variance accounted for by movementtype manipulation were $48 \%$ and $24 \%$, respectively. The BSC tests were performed with a significantly larger degree of angle error and significantly more slowly than were the within (SF and WSC) tests. Performances on SF and WSC tests were not significantly different.

Although the "between" tests were less accurate than both "within" tests, the "between" movements were more accurate than the level predicted by chance, which is $90 \mathrm{deg}$. The mean angle error of "between" movements on problems when the two paths were learned $(54.0 \mathrm{deg})$ was significantly less than the mean angle error of "between" movements on problems when the subjects were required to guess $[85.6 \mathrm{deg} ; \mathrm{t}(11)=$ $3.75, \mathrm{p}<.05]$. This guessing value, which was obtained from trials on which the subjects did not leam the two paths, is not significantly different from chance $[t(11)=$ $.51, \mathrm{p}>.05]$.

The mean thinking RTs for problems tested with each type of movement did not differ significantly $[F(2,22)<1.0, p>.05]$. This suggests that the subjects performed the integration phase of the problem at a consistent rate for each test. None of the Pearson correlation coefficients for the simple forward shortcuts within one path and the shortcuts between two paths of the subjects' mean angle errors, drawing RTs, and thinking RTs were significantly greater than zero at the .05 level.

Effects of training. It was anticipated that the hightraining procedure would have reduced the overall difficulty of integrating the paths and performing the tests. Doubling the amount of practice should have increased the subjects' familiarity with the paths and led to improved performance. However, the different amounts of training did not produce any significant effects. The mean angle errors for the high- and lowtraining conditions were $36.4 \mathrm{deg}$ vs. $40.1 \mathrm{deg}$, respectively $[F(1,11)=3.08, p>.05]$, the mean drawing RTs were $9.9 \mathrm{sec}$ vs. $8.3 \mathrm{sec}$, respectively $[\mathrm{F}(1,11)=3.86$, $\mathrm{p}>.05]$, and the mean thinking RTs were $8.0 \mathrm{sec}$ vs. $7.4 \sec [F(1,11)<1.0, p>.05]$. None of the training $x$ movement interactions for the three dependent variables were significant.

\section{Discussion}

Simple forward movements and shortcuts within one path were performed with the same speed and accuracy. This finding replicates those of Levine et al. (1982). However, the data did not support the hypothesis that the integrated representation of the two component paths was simultaneous, with all spatial relationships equally available. Inferred movements between the two paths were performed with less accuracy and more slowly than were either originally learned or inferred movements within one path. Nevertheless, there was some evidence that the integrated path was represented. The subjects performed more accurately than if they had just been guessing on the BSC movements. The data suggest that subjects could integrate independently learned paths but that the structure of the integrated representation was inaccurate.

One possible explanation of these data is that cognitive integration could generate a simultaneous representation of the combined path but that some segments of the two paths were poorly learned or forgotten. The subjects may not have been able to maintain a complete representation in memory. To perform a "within" movement, the subjects had to remember only one path. On the other hand, shortcuts between two paths required the subjects to remember both paths. Consider, for example, the extreme case in which subjects always remembered only a simple path. On 50\% of the "within" tests, they were tested on the remembered path and performed well. On the remaining 50\%, they were forced to guess. However, on $100 \%$ of the "between" tests, they were forced to guess, since knowledge of a single path was not sufficient to infer the proper movement. In general, forgetting one path impaired the overall "within"-movement performance less than it did the "between"-movement performance. This possibility that the subjects forgot spatial information implies that equiavailability of spatial relationships should be found only if the probability of recalling both paths is high. This hypothesis could be tested by giving the subjects more effective practice and by assessing their memory for the paths.

\section{EXPERIMENT 2}

In order to ensure better memory of the paths, a few procedural changes were instituted. The subjects received four consecutive tracings on each path. They could then request additional tracings on either or both paths until they felt they knew each path very well. Furthermore, in order to determine whether the training was effective, the subjects were asked at the end of each problem to rate how well they remembered the path on which they had been tested. We chose this procedure rather than one of training all subjects to some performance criterion because we anticipated that permitting the subjects to define their own criteria of 
knowing information well would be sufficient. Our hypothesis was that if all the path information was recalled (i.e., high memory ratings were given), then equiavailability should occur: both "between"- and "within"-movement types should be equally accurate. When the path information was partially recalled (i.e., intermediate memory ratings were given), equiavailability might not occur. When low memory ratings were given, all the movements should be performed very inaccurately, because all or most of the path information would not be remembered.

\section{Method}

Subjects. Thirty right-handed undergraduates from the State University of New York at Stony Brook fulfilled a course requirement by participating in the experiment.

Procedure. Essentially the same stimuli and procedure from Experiment 1 were used. There were, however, a few changes. All subjects had each path traced four consecutive times. Once the standard amount of tracing had been completed, the subjects could request additional tracings on either or both paths until they felt they remembered each path very well. The number of additional tracings was not recorded. Fifteen subjects always had the numbered path traced first, and the other 15 subjects always had the lettered path traced first. No mention was made of the general relationship between Points 1 and 3 and Points A and $\mathrm{C}$.

The subjects were instructed to say when they had had sufficient training on both paths. After their signal, an integration point was announced. The subjects could not receive any additional tracings after the integration point had been given. As in Experiment 1, the subjects received one of three tests: a "within" test (SF or WSC) or a "between" test (BSC). After performing a test movement, the subjects were asked to rate (on a scale of 1 to 10) how well they remembered the path on which they had been tested. A rating of 1 corresponded to no memory of the path at all, and 10 corresponded to remembering the path perfectly. If the test had been a "within" movement, the subjects were instructed to rate only their memory for the single three-point path that contained the tested relation. For example, after having moved from Point 1 to Point 3 , they were asked to rate the 1-2-3 path. If the test movement had been a "between" movement, the subjects were asked to rate the entire combined path. The memory rating was recorded, and the angle error of the test movement was measured for each problem. All subjects were presented with 15 problems in a different random order. The type of movement on each problem was counterbalanced across subjects. The 15 experimental problems were the same for all subjects and were preceded by 3 practice problems. Within these 18 problems, all nine integration points were presented twice in a single 1-h session. The subjects never received any feedback, whether verbal or visual, from the experimenter about their performance throughout the experiment.

\section{Results}

The data were analyzed using a repeated-measures analysis of variance. All paired comparisons found to be significant in the overall analysis of variance were analyzed with the Newman-Keuls test. Six subjects requested a second attempt on a single trial, and one subject requested second attempts on three different trials. In all, seven of the nine second attempts were more accurate than were the first movements.

Effects of type of movement. As shown in Table 2, "between" movements were again performed with
Table 2

The Means and Standard Deviations of Angle Errors (in Degrees) and Memory Ratings ( 1 to 10 ) for the Test Movements

\begin{tabular}{cccccc}
\hline \multirow{2}{*}{$\begin{array}{c}\text { Test } \\
\text { Movement }\end{array}$} & \multicolumn{2}{c}{ Angle Error } & & \multicolumn{2}{c}{ Memory Rating } \\
\cline { 2 - 3 } \cline { 5 - 6 } & Mean & SD & & Mean & SD \\
\hline SD & 32.10 & 17.98 & & 6.50 & 1.48 \\
WSC & 29.00 & 14.82 & & 6.40 & 1.30 \\
BSC & 51.60 & 27.28 & & 5.50 & 1.68 \\
\hline
\end{tabular}

Note-SF = simple forward; $W S C=$ shortcut within one path; $B S C=$ shortcut between two paths.

significantly less accuracy than were both "within" movements $[F(2,58)=10.63, p<.05]$. The movementtype effect accounted for $19 \%$ of the variance. There was no significant difference in angle error between SF and WSC tests. The generalized version of the equiavailability principle again appeared to be unsupported. Both memory ratings for the within-path tests were, however, significantly higher than were the memory ratings of the between-path tests $[\mathrm{F}(2,58)=11.68$, $p<.05]$. Ten percent of the variance was accounted for by this effect. Since the equiavailability hypothesis may have been applicable only when the information was well remembered, a more thorough analysis of the memory ratings was required.

Memory ratings of the paths. Before the memoryrating results were used more analytically, the construct validity of the ratings had to be evaluated. For each of the three types of movements, the subjects' mean angle errors were significantly correlated with their memory ratings. The Spearman correlation coefficients were $-.48,-.34$, and -.41 for the SF, WSC, and BSC tests, respectively. As the subjects' judged knowledge of the tested path increased, the movement error decreased.

We next categorized the problems according to how well the subjects indicated that they remembered the paths. Five categories were used: memory ratings of 1 and 2, 3 and 4, 5 and 6,7 and 8 , and 9 and 10 . The overall mean angle errors of movements were 70.5, 54.1, $43.5,27.8$, and $12.7 \mathrm{deg}$, respectively, when the individual problems were grouped into the five rating categories. ${ }^{1}$ Memory ratings of $1 \mathrm{~s}$ and $2 \mathrm{~s}$ corresponded to very inaccurate movements. At the other extreme, for the highest memory ratings ( $9 \mathrm{~s}$ and $10 \mathrm{~s}$ ), the movements were very inaccurate. These results indicate the validity of the memory ratings as indices of the subjects' ability to remember the path information and locate the target.

It may be argued that since the subjects were required to rate the quality of their memory after they had drawn their lines, the memory ratings could have only reflected the subjects' confidence in their responses. But how do subjects arrive at a confidence rating when they have never been given any feedback about their responses on a task that was novel and when the tested information continually and nonsystematically changed? To a large degree, the subjects' confidence must have been based on how well they remembered the tested informa- 
tion, which is what we have interpreted the memory ratings to roughly represent.

Therefore, given that performance is impaired by forgetting, we next sought to determine directly whether the inferiority of BSC movements was a result of forgetting. To put it another way, does equiavailability appear when all the component-path information is well learned and remembered? The performances on problems in which the subjects recalled the path information equally well were contrasted. The mean "between" and "within" performances were compared for each of the five rating categories. The results are shown in Figure 3. The Bonferroni t statistic was used to test the significance of these paired comparisons. ${ }^{2}$ When most or all of the path information was remembered (memory ratings of $7-8$ and 9-10), "between" movements were performed with the same high accuracy as "within" movements [for 7.8, $\mathrm{t}(150)=1.01, \mathrm{p}>.05$; for 9.10 , $t(53)=.78, p>.05]$. "Between" tests were also as accurate as "within" movements when the memory ratings were very low [for $1-2, \mathrm{t}(28)=-.08, \mathrm{p}>.05]$. However, when an intermediate amount of information was recalled, that is, for memory ratings of 3.4 and 5-6, movements within one path were significantly more accurate than were movements between two paths [for $3-4, t(56)=2.30, p<.05 ;$ for $5-6, t(153)=3.89$, $\mathrm{p}<.05]$.

Individual differences were examined to address some problems with the above analysis. The equiavailability hypothesis predicts equal accuracy, which in turn forces one to accept the null hypothesis. If the differences between the within-path and betweenpath movements were due to the subjects' memory of the tested information, a predictable pattern of results should occur. The subjects who gave a higher overall memory rating on the "between" tests than on the "within" tests should have performed the "between" movements more accurately, not less accurately, than they did the "within" movements. If the subjects" overall memory ratings for both types of tests were the same, then their performances should have been the same. Finally, if the "within"-test memory ratings were higher than the "between"-test memory ratings, "within" movements should have been more accurate than "between" movements.

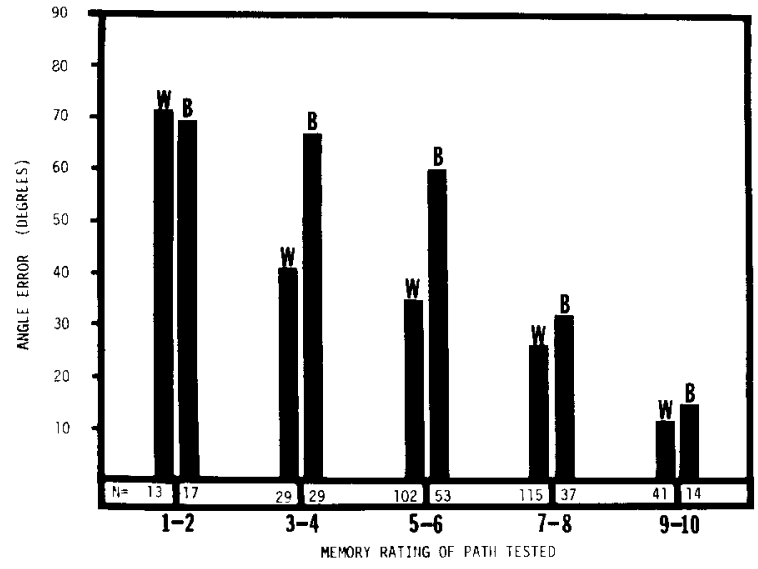

Figure 3. The mean angle error of movements within one path $(W)$ and movements between two paths (B) within each memory-rating category.

The mean difference in memory ratings (DMR) for "between" tests and "within" tests was computed for each subject. The subjects were placed in one of five groups generated by the following criteria: (1) 1.5 $\geqslant$ DMR $>0.5$, (2) $0.5 \geqslant$ DMR $>-0.5$, (3) $-0.5 \geqslant$ DMR $>-1.5$, (4) $-1.5 \geqslant$ DMR $>-2.5$, and (5) -2.5 $\geqslant$ DMR $>-3.5$. Table 3 shows the mean angle errors and memory ratings of "between" and "within" tests for subjects blocked by the difference between their mean memory ratings. The pattern of response accuracy parallels the above predictions. In particular, "between" movements were more accurate than "within" movements when the subjects' overall memory ratings for tests on integrated paths were greater than their overall memory ratings for tests on single, three-point paths. Table 3 also indicates that the subjects differed in their mean memory ratings for the "between" tests and not for the "within" tests. All subjects performed the "within" movements with about the same accuracy and rated their memory for the single-path tests about the same. This suggests that all subjects were able to remember and use the single paths equally well, but that they differed in their abilities to generate and remember the integrated representations.

Effects of training. Allowing the subjects to receive additional training up to their own criterion did not

Table 3

The Means and Standard Deviations of Angle Errors (in Degrees) and Memory Ratings ( 1 to 10) for Between and Within Tests After Subjects Were Blocked by the Difference Between Their Mean Memory Ratings (Between Memory Rating-Within Memory Rating)

\begin{tabular}{|c|c|c|c|c|c|c|c|c|c|}
\hline \multirow{3}{*}{$\begin{array}{c}\text { Difference } \\
\text { Between Mean } \\
\text { Memory Ratings }\end{array}$} & \multirow[b]{3}{*}{$\mathrm{n}$} & \multicolumn{4}{|c|}{ Angle Error } & \multicolumn{4}{|c|}{ Memory Rating } \\
\hline & & \multicolumn{2}{|c|}{ Between } & \multicolumn{2}{|c|}{ Within } & \multicolumn{2}{|c|}{ Between } & \multicolumn{2}{|c|}{ Within } \\
\hline & & Mean & SD & Mean & SD & Mean & SD & Mean & SD \\
\hline 1.0 & 3 & 22.30 & 4.80 & 31.90 & 8.11 & 7.90 & 0.61 & 7.10 & 0.64 \\
\hline 0.0 & 6 & 32.80 & 13.12 & 34.50 & 7.57 & 6.20 & 1.64 & 6.30 & 1.60 \\
\hline-1.0 & 12 & 60.00 & 33.41 & 32.50 & 16.15 & 5.20 & 1.42 & 6.20 & .1 .29 \\
\hline-2.0 & 6 & 59.90 & 17.26 & 25.10 & 9.66 & 5.20 & 0.86 & 7.20 & 0.90 \\
\hline-3.0 & 3 & 67.30 & 11.22 & 28.80 & 14.90 & 3.10 & 0.64 & 6.00 & 0.55 \\
\hline
\end{tabular}


improve movement accuracy as anticipated. Table 4 shows the mean angle errors for the three types of test movements under the ad-lib training condition of Experiment 2 and the low-training (two tracings) and the high-training (four tracings) conditions of Experiment 1 . Included in Table 4 are some data from another study that used the same stimulus materials and very similar procedures to those used in Experiment 2. The subjects in this experiment received four consecutive tracings over each path twice (a total of eight tracings over each path). A $4 \times 3$ analysis of variance tested whether the amount of initial training produced a significant effect on movement accuracy; the amount of training was a between factor and the type of movement was a within factor. There were no significant differences in angle error among the four amounts of training $[F(3,59)<1.0, p>.05]$, a significant main effect of movement type $[F(2,118)=27.81, p<.05]$, and no significant interaction $[F(6,118)<1.0, p>.05]$. The effect of movement type accounted for $23 \%$ of the variance. Tracing each path two, four, or eight times did not affect the accuracy of the movements. Even when the subjects themselves requested additional training until they knew each path very well before integration, the movement accuracy did not improve.

\section{Discussion}

The originally learned and inferred movements within a single path were equally accurate, as in Experiment 1 and as Levine et al. (1982) reported. Their hypothesis that a sequentially traced path is simultaneously represented and that all relationships are consequently equally available was supported. We also found that movements within one path were often more accurate than were movements between two paths, even when the subjects were allowed to become very familiar with the individual paths. The integrated layouts appeared not to be consistently represented with equally accurate relationships between paths and within paths.

\section{GENERAL DISCUSSION}

The performances on the cognitively integrated paths in the present experiments can be compared with performances on the same-size paths that were organized into a single region during acquisition. Levine et al. (1982, Experiment 2) traced blindfolded subjects' fingers over five-point paths (which contained the same number of segments as the integrated paths) in a continuous sequence. Under these learning conditions, the equiavailability principle was confirmed: Subjects moved forward or in reverse along originally learned and inferred paths with equivalent accuracy. The inferred movements in the Levine et al. experiment corresponded to the movements between two paths in the present experiments (simple forward movements were the same type of test in both experiments). A working hypothesis about survey knowledge has been that survey knowledge is independent of the way the spatial layout is learned. The results from the present experiments do not support this hypothesis. The separate acquisition and subsequent integration of the component regions affected the accuracy of the subjects' survey knowledge of the integrated layouts.

Although independent acquisition and subsequent integration can influence a person's survey knowledge, these processes do not necessarily determine the cognitive organization of the environment. We have demonstrated that when path information is well remembered, the subjects are not only able to cognitively integrate independently learned parts of an environment, but also show equiavailability, that is, take shortcuts between paths as accurately as within paths. The data on individual differences suggest that some subjects had the cognitive skills needed to overcome the effects of independently learning the paths.

A confirmation of the equiavailability predictions was presented recently by Moar and Carleton (1982). They investigated the acquisition of two intersecting routes through a large-scale environment. Subjects viewed one sequence of slides that simulated a walk through an urban area and then immediately viewed a second sequence of slides depicting a walk along a different route through the same region. Large segments of the intersecting routes were the same, thus enabling subjects to spatially relate the segments of the different paths while learning the layouts. That is, subjects could integrate the two paths during the acquisition of the second path. Moar and Carleton found that subjects could locate points within the same route and in differ-

Table 4

The Means and Standard Deviations of Angle Errors (in Degrees) for the Three Test Movements When Subjects Received Two, Four, Eight, or Ad-Lib Tracings on Each Path

\begin{tabular}{|c|c|c|c|c|c|c|c|}
\hline \multirow{3}{*}{$\begin{array}{l}\text { Number } \\
\text { of Tracings }\end{array}$} & \multirow[b]{3}{*}{$\mathrm{n}$} & \multicolumn{6}{|c|}{ Test Movement } \\
\hline & & \multicolumn{2}{|c|}{ SF } & \multicolumn{2}{|c|}{ WSC } & \multicolumn{2}{|c|}{ BSC } \\
\hline & & Mean & SD & Mean & SD & Mean & $S D$ \\
\hline Two & 12 & 37.20 & 15.92 & 30.90 & 7.06 & 50.90 & 13.72 \\
\hline Four & 12 & 24.60 & 9.42 & 27.90 & 10.21 & 56.10 & 8.13 \\
\hline Eight & 9 & 30.70 & 23.00 & 34.80 & 20.24 & 57.80 & 29.48 \\
\hline Ad Lib & 30 & 32.10 & 17.98 & 29.00 & 14.82 & 51.60 & 27.28 \\
\hline
\end{tabular}

Note $-S F=$ simple forward; $W S C=$ shortcut within one path; BSC = shortcut between two paths. 
ent routes equally well, suggesting that the two paths were integrated into a single cognitive map (i.e., that the relationships between paths and within paths were equally available). These learning conditions were similar to those in the Levine et al. experiments, in which subjects could integrate all the path segments during acquisition because they were presented with the layout in a continuous sequence. Moar and Carleton also found that the repeated presentation of the routes improved the accuracy of the subjects' spatial judgments.

This finding is quite different from the present results for the effects of training. An account of this difference might be that subjects in the Moar and Carleton studies had many opportunities to integrate the paths more accurately and completely with the repeated presentation of the routes. In the present experiments, the repeated tracing of the individual paths only gave the subjects the opportunity to learn the separate paths, because the subjects could only integrate the layouts after the acquisition of the spatial information. In conclusion, the time at which integration occurs during the learning of a spatial layout appears to be influential in the acquisition and accuracy of survey knowledge.

\section{REFERENCE NOTE}

1. Shemyakin, F. N. Orientation in space. In B. G. Ananyev, G. S. Kostyuk, A. N. Leontyev, A. R. Luria, M. A. Menchinskaya, S. L. Rubinshteyn, A. A. Smirnov, B. M. Teplov, \& F. N. Shemyakin (Eds.), Psychological science in the USSR (Vol. 1) (Tech. Rep. 11466, Pt. 1). Washington, D.C: U.S. Office of Technical Reports, 1962.

\section{REFERENCES}

APPLEYARD, D. Styles and methods of structuring a city. Environment and Behavior, 1970, 2, 100-118.

Chase, W. G., \& Chi, M. T. H. Cognitive skill: Implications for spatial skill in large-scale environments. In J. Harvey (Ed.), Cognition, social behavior and the environment. Potomac, MD: Erlbaum, 1980.
Downs, R. M. Maps and mapping as metaphors for spatial representation. In L. S. Liben, A. H. Patterson, \& N. Newcombe (Eds.), Spatial representation and behavior across the life span: Theory and application. New York: Academic Press, 1981.

Downs, R. N., \& SteA, D. Image and environment: Cognitive mapping and spatial behavior. Chicago: Aldine, 1973.

Levine, M., Jankovic, I. N., \& Palis, M. Principles of spatial problem solving. Journal of Experimental Psychology: General, $1982,111,157-175$.

LYNCH, K. The image of the city. Cambridge, Mass: M.I.T. Press, 1960.

Moar, I., \& Carleton, L. R. Memory for routes. Quarterly Journal of Experimental Psychology, 1982, 34A, 381-394.

MYERS, J. L. Fundamentals of experimental design (3rd edition). Boston: Allyn \& Bacon, 1979.

Siegel, A. W. The externalization of cognitive maps by children and adults: In search of ways to ask better questions. In L. S. Liben, A. H. Patterson, \& N. Newcombe (Eds.), Spatial representation and behavior across the life span: Theory and applica. tion. New York: Academic Press, 1981.

Sieget, A. W., Kirasic, K. C., \& KaIl, R. V. Stalking the elusive cognitive map: The development of children's representations of geographic space. In J. F. Wohlwill \& I. Altman (Eds.), Human behavior and environment (Vol. 3). New York: Plenum, 1978.

Siegel, A. W., \& White, W. H. The development of spatial representations of large-scale environments. In $\mathbf{H}$. W. Reese (Ed.), Advances in child development and behavior (Vol. 10). New York: Academic Press, 1975.

Tolman, E. C. Cognitive maps in rats and men. Psychological Review, 1948, 55, 189-208.

\section{NOTES}

1. The decrease in mean absolute angle errors was not due to systematic biases in movements, because the variability in angle error also decreased as the memory ratings increased. If systematic movement biases were responsible for the progression of angle errors, the variability in angle error would not have continually decreased from an almost random level of performance to a very accurate level. The standard deviations of angle error were $49.53,45.24,40.39,32.30$, and $12.89 \mathrm{deg}$ for the $1-2$ to 9-10 memory-rating categories, respectively.

2. The Bonferroni $t$ is a test statistic for nonorthogonal contrasts (Myers, 1979) and is appropriate for these comparisons.

(Manuscript received October 12, 1982; revision accepted for publication April 8, 1983.)

\section{4th ANNUAL MEETING OF THE PSYCHONOMIC SOCIETY, INC.- CORRECTED DATES}

\begin{abstract}
Announcement-24th Annual Meeting of The Psychonomic Society, Inc. (Bulletin of the Psychonomic Society, 1983, 21, 324; Perception \& Psychophysics, 1983, 34, 193)-Please note that the wrong dates for the 1983 meeting were provided. The correct dates for this year's meeting are those on the Program, November 17-19, 1983.
\end{abstract}

